\title{
WEAK HIRONAKA THEOREM
}

\section{Fedor A. Bogomolov and Tony G. Pantev}

\section{Introduction}

The existence of a smooth projective model for any proper algebraic variety over an algebraically closed field of characteristic zero is one of the most important results in algebraic geometry.

H.Hironaka [Hi] proved a very strong version of the above statement. For any such variety he established the existence of a sequence of blow ups which resolve the singularities of the variety. Moreover at every step the blow ups occur along smooth subvarieties of the singular locus. In particular the process does not modify the nonsingular part of the initial variety. This result proved to be even more useful than the existence of a smooth model, but its proof was rather complicated. Later on, E. Bierstone and P. Milman [B-M] and M. Spivakovsky [S] found different versions of the resolution process that are considerably simpler and canonical but somehow in principle their proofs follow the same track.

In this note we want to give a proof of the existence of a smooth projective model for any variety in characteristic zero using an idea similar to A.J. de Jong's approach in $[\mathrm{dJ}]$. We present a straightforward way to obtain a nonsingular model which however does not permit any control over the intermediate steps.

The main result of this note is the following theorem

Theorem A. Let $X$ be a normal projective variety over an algebraically closed field $k$, char $k=0$ and let $D \subset X$ be a proper subvariety of $X$. Then there exist a smooth projective variety $M$, a strict normal crossings divisor $R \subset M$ and a birational morphism $f: M \rightarrow X$ with $f^{-1} D=R$

Our proof uses induction on the dimension of $X$. It is organized as follows. In section 2 we construct a finite morphism $(\widehat{X}, \widehat{D}) \rightarrow\left(P, S_{0}\right)$ from a suitable blow up $(\widehat{X}, \widehat{D})$ of the pair $(X, D)$ to a pair $\left(P, S_{0}\right)$ where $P$ is a compactification of the total space of a line bundle over $\mathbb{P}^{n-1}, S_{0}$ is the image of a section of this line bundle and $\widehat{X} \rightarrow P$ is branched over a divisor $B=\sum S_{i}$ which is a sum of distinct sections of $P \rightarrow \mathbb{P}^{n-1}$. After this is

Received April 3, 1996.

The first author is partially supported by NSF grant DMS-9500774.

The second author is partially supported by NSF grant DMS-9500712. 
achieved we proceed in section 3 by applying the induction hypothesis to the pair $\left(\mathbb{P}^{n-1}, Z\right)$. Here $Z \subset \mathbb{P}^{n-1}$ is the discriminant locus of the set of sections $\left\{S_{i}\right\}$, i.e. $Z$ is the unique closed reduced subscheme in $\mathbb{P}^{n-1}$ whose preimage in $P$ contains all possible intersections of the $S_{i}$ 's. By induction we can find a birational morphism $\widetilde{\mathbb{P}}^{n-1} \rightarrow \mathbb{P}^{n-1}$ which transforms $Z$ into a strict normal crossings divisor $\widetilde{Z}$. Put $\widetilde{P}$ for the variety obtained from $P$ via a base change with $\widetilde{\mathbb{P}}^{n-1} \rightarrow \mathbb{P}^{n-1}$. Then $\left(\widetilde{P} \rightarrow \widetilde{\mathbb{P}}^{n-1},\left\{S_{i}\right\}\right)$ is a family of pointed $\mathbb{P}^{1}$ 's and an application of $\mathrm{F}$. Knudsen's stabilization theorem yields a smooth variety $Q$ equipped with a contraction map onto $\widetilde{P}$ and such that the complete preimage of $B$ in $Q$ is a normal crossings divisor. Now the fiber product of $Q$ and $\widehat{X}$ has only abelian quotient singularities which can be resolved by a toroidal blow-up. The resulting pair $(\mathrm{M}, \mathrm{R})$ will be the resolution of $(X, D)$.

Remark 1.1. Recently A.J. de Jong [dJ] invented a remarkable new approach which allowed him to resolve in any characteristic at least some variety which dominates the initial variety by a finite map. The idea to fiber $X$ by curves and use induction on the dimension came from de Jong's insight in $[\mathrm{dJ}]$. The important reduction in section 2 imitates the first part of the proof of the famous theorem of Belyi [B].

Remark 1.2. The fact that $\operatorname{char} k=0$ is used only at last step of the argument in section 3 since in the case of a positive characteristic it is hard to control the unramified coverings of the torus.

Remark 1.3. A different proof of the same theorem was found independently by D. Abramovich and A.J. de Jong [A-dJ]. It uses similar ingredients but is quite different in detail. In particular their proof has the advantage of being better adapted to handling equivariant resolutions.

\section{The map to a $\mathbb{P}^{1}$ bundle}

Let $X$ be an irreducible variety of dimension $n$ over $k$ and let $D \subset X$ be a closed subvariety. The aim of this section is to prove the following theorem.

Theorem 2.1. There exists a finite morphism $(\widehat{X}, \widehat{D}) \rightarrow\left(P, S_{0}\right)$ from a suitable blow up $(\widehat{X}, \widehat{D})$ of the pair $(X, D)$ to a pair $\left(P, S_{0}\right)$ of varieties satisfying:

(i) $P=\mathbb{P}(\mathcal{O} \oplus L)$ for some line bundle $L \rightarrow \mathbb{P}^{n-1}$.

(ii) $S_{0} \subset P$ is the image of a section of $L$.

(iii) The finite morphism $\widehat{X} \rightarrow P$ is branched over a divisor $B$ of the form

$$
B=\sum_{i=1}^{k} S_{i}+S_{k+1}
$$


where the $S_{i}$ 's, $i=1, \ldots, k$ are images of distinct sections of $L$ and $S_{k+1}:=\mathbb{P}_{\infty}$ is the infinity section of $P \rightarrow \mathbb{P}_{n-1}$.

Proof. Passing to a blow up of $(X, D)$ if necessary we may assume without loss of generality that $X$ is normal and that $D=\sum D_{i}$ with $D_{i}$ reduced and irreducible divisors. We start with the following relative version of the Noether normalization lemma.

Lemma 2.2. There exists a finite morphism $f: X \rightarrow \mathbb{P}^{n}$ which maps $\widetilde{D}$ onto a hyperplane $\mathbb{P}^{n-1}$ of $\mathbb{P}^{n}$.

Proof. In order to find such a map it is enough to consider the embedding of $X$ into projective space via a very ample line bundle which has a section vanishing on every $D_{i}$. This can be easily achieved by taking a sufficiently big line bundle. Let $X \subset \mathbb{P}^{m}$ be such an embedding and let $H \subset \mathbb{P}^{m}$ be the hyperplane containing all $D_{i}$. A straightforward dimension count shows that the generic subspace $\mathbb{P}^{N-n-1} \subset H$ does not intersect $X \cap H$ and therefore we can take for $f$ the projection of $\mathbb{P}^{m}$ centered at such a subspace.

Lemma 2.3. The map $f$ can be chosen so that there exists a point $o \in \mathbb{P}^{n}$ satisfying the following properties:

(a) the preimage of o consists of a finite number of smooth points $x_{i}$ with $d f_{x_{i}}$ being an isomorphism for every $x_{i}$.

(b) the preimage of every line in $\mathbb{P}^{n}$ containing o is a connected and generically reduced curve.

(c) the preimage of the generic line trough o is irreducible.

Proof. The subset of points satisfying (a) is open in $\mathbb{P}^{n}$ due to the generic smoothness lemma. In order to satisfy (b) it suffices to chose the projective embedding from lemma 2.2 in such a way that all non-reduced curves in $X$ obtained as hyperplane sections constitute a subvariety of big codimension (greater than $n-1$ ) in the corresponding Grassmanian. In other words we want to choose the embedding $X \subset \mathbb{P}^{m}$ so that there exists a projective subspace $V \subset \mathbb{P}^{m}$ of dimension $m-n$ with the property

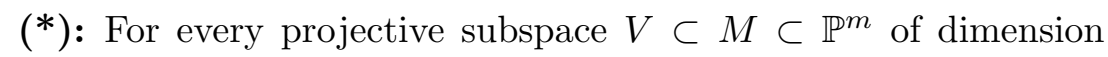
$m-n+1$ the intersection $M \cap X$ is a generically reduced connected curve.

Due to the connectedness theorem [Ha, III Corollary 7.9], [F-H] the preimage in $X$ of every line trough $o$ will be connected. Let $Y$ be the union of the singular locus of $X$ the divisor $D$ and the ramification divisor of $f$. Furthermore Bertini's theorem implies that the part of a general hyperplane curve contained in $X \backslash Y$ will be reduced and irreducible and therefore it 
suffices to show that for a suitable embedding of $X$ there exists a $m-n$ dimensional projective subspace $V \subset \mathbb{P}^{m}$ satisfying

$(* *)$ : For every projective subspace $V \subset M \subset \mathbb{P}^{m}$ of dimension $m-n+1$ the intersection $M \cap Y$ is of dimension zero.

Consider the dimension jump locus

$\Gamma=\{M \mid \operatorname{dim} M=m-n+1, \operatorname{dim}(M \cap Y) \geq 1\} \subset G r(m-n+2, m+1)$,

and let $\widetilde{\Gamma}=\{(M, y) \mid y \in M\} \subset \Gamma \times Y$ be the corresponding incidence variety. The projection $\pi_{Y}: \widetilde{\Gamma} \rightarrow Y$ surjects on $Y$ and its fiber over a general point $y \in Y$ consists of all $M \subset \mathbb{P}^{m}$ of dimension $m-n+1$ such that $y \in M$ and $\operatorname{dim}(M \cap Y) \geq 1$. In particular $\operatorname{dim} \widetilde{\Gamma} \leq 2 n-3$ and $\operatorname{dim} \Gamma \leq 2 n-4$. Furthermore the flag variety $F$ parametrizing flags $V \subset M \subset \mathbb{P}^{m}$ as above has the usual double fibration structure

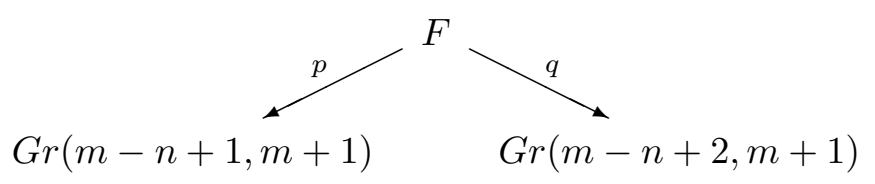

where the fibers of $p$ are projective spaces of dimension $n-1$ and the fibers of $q$ are projective spaces of dimension $m-n$. The locus in $G r(m-n+1, m+1)$ consisting of all $V$ 's that do not satisfy (**) is contained in $q\left(p^{-1}(\Gamma)\right)$ and hence has codimension that is $\geq(m-n+$ 1) $n-(m-n+2 n-4)=m(n+1)-n^{2}+4$. Thus composing our original embedding $X \subset \mathbb{P}^{m}$ with a Veronese embedding of sufficiently high degree we can make this codimension strictly positive which proves the lemma.

Denote by $P_{1}$ the blow up of $\mathbb{P}^{n}$ at $o$ and by $\widehat{X}$ the blow up of $X$ at the points $x_{i}$. Since the blow up at $o \in \mathbb{P}^{n}$ resolves the projection centered at $o$ we get a realization of $P_{1}$ as a $\mathbb{P}^{1}$ bundle over $\mathbb{P}^{n-1}$. There is a canonical isomorphism $P_{1} \cong \mathbb{P}\left(\mathcal{O}_{\mathbb{P}^{n-1}} \oplus \mathcal{O}_{\mathbb{P}^{n-1}}(1)\right)$ which gives a natural tautological line bundle $\mathcal{O}_{P_{1}}(1) \rightarrow P_{1}$ and the preimage $\mathbb{P}_{\infty}$ of $o$ in $P_{1}$ is a section of $\mathcal{O}_{P_{1}}(1)$. Alternatively, $\mathbb{P}_{\infty}$ is the divisor in $\mathbb{P}\left(\mathcal{O}_{\mathbb{P}^{n-1}} \oplus \mathcal{O}_{\mathbb{P}^{n-1}}(1)\right)$ corresponding to the line subbundle $\mathcal{O}_{\mathbb{P}^{n-1}} \subset \mathcal{O}_{\mathbb{P}^{n-1}} \oplus \mathcal{O}_{\mathbb{P}^{n-1}}(1)$ and $P_{1}$ can be thought of as a compactification of the total space of $\mathcal{O}_{\mathbb{P}^{n-1}}(1)$ with the divisor $\mathbb{P}_{\infty}$ at infinity.

By construction $\widehat{X}$ is fibered by connected and generically reduced curves $C_{t}, t \in \mathbb{P}^{n-1}$ and maps fiberwise via $f$ to the $\mathbb{P}^{1}$-fibration $P_{1}$. Due to the smoothness of $P_{1}$ the Zariski-Nagata purity theorem [SGA 1, X 3.1] implies that the branch locus $B_{1}$ of the finite map $f: \widehat{X} \rightarrow P_{1}$ is a divisor. Moreover, since $C_{t}$ is generically reduced for every $t$ we conclude that $B_{1}$ is a horizontal divisor in $\pi: P_{1} \rightarrow \mathbb{P}^{n-1}$, i.e. every component of $B_{1}$ is 
mapped finitelyonto $\mathbb{P}^{n-1}$ by $\pi$. Denote by $N$ the degree of $B_{1}$ on the fibers of $\pi$. Now we can apply Belyi's construction to simplify the branch divisor.

We will need the following technical result:

Lemma 2.4. Let $L$ be a line bundle on a variety $M$ and $B$ be a horizontal divisor in the total space tot $(L)$ of $L$, which maps properly on $M$ under a natural projection $\pi: \operatorname{tot}(L) \rightarrow M$. Denote by d the degree of the finite map $\pi: B \rightarrow M$. There exists a canonical fiberwise morphism $p_{B}: \operatorname{tot}(L) \rightarrow$ $\operatorname{tot}\left(L^{\otimes d}\right)$ which is polynomial of degree $d$ on any fiber $L_{x}, x \in M$ and such that $B$ is the preimage of the zero section of $L^{\otimes d}$ under $p_{B}$. The map $p_{B}$ is defined uniquely modulo multiplication by invertible functions on $M$.

Proof. Consider the compactification $P_{L}:=\mathbb{P}\left(\mathcal{O}_{M} \oplus L\right)=\operatorname{Proj}\left(\operatorname{Symm}{ }^{\bullet}\left(\mathcal{O}_{M}\right.\right.$ $\left.\left.\oplus L^{-1}\right)\right)$. Let $M_{\infty}$ be the infinity section of $P_{L}$ and $y \in H^{0}\left(M, \mathcal{O}_{M}\right) \subset$ $H^{0}\left(P_{L}, \mathcal{O}_{P_{L}}(1)\right)$ be a non-zero element. Then $\operatorname{div}(y)=M_{\infty}$. Denote by $\lambda \in H^{0}\left(\operatorname{tot}(L), \pi^{*} L\right)$ the tautological section and let $x \in H^{0}\left(P_{L}, \mathcal{O}_{P_{L}}(1) \otimes\right.$ $\left.\pi^{*} L\right)$ be the section whose divisor is the zero section of $L$ normalized so that $\lambda=x / y$. The fact that the intersection of $B$ with the generic fiber of $\pi: P_{L} \rightarrow M$ is $d$ implies that $\mathcal{O}_{P_{L}}(B)=\mathcal{O}_{P_{L}}(d) \otimes \pi^{*} A$ for some line bundle $A$ on $M$. Moreover since $B \subset \operatorname{tot}(L)$ it follows that $\mathcal{O}(B)_{\mid M_{\infty}}=\mathcal{O}$ and hence $\pi^{*} A_{\mid M_{\infty}} \cong \mathcal{O}_{P_{L}}(-d)_{\mid M_{\infty}}$. On the other hand $P_{L}=\mathbb{P}\left(\mathcal{O}_{M} \oplus L\right)$ and by using that $\pi_{\mid M_{\infty}}$ is an isomorphism we get that $A=L^{\otimes d}$.

Let $\varphi \in H^{0}\left(P_{L}, \mathcal{O}_{P_{L}}(d) \otimes \pi^{*} L^{\otimes d}\right)$ be a section with divisor $B$. Applying the projection formula to the pushforward by $\pi$ we get isomorphisms

$$
\begin{aligned}
& H^{0}\left(P_{L}, \mathcal{O}_{P_{L}}(d) \otimes \pi^{*} L^{\otimes d}\right)=H^{0}\left(M,\left(\pi_{*} \mathcal{O}_{P_{L}}(d)\right) \otimes L^{\otimes d}\right)= \\
& H^{0}\left(M, S^{d}\left(\mathcal{O}_{M} \oplus L^{-1}\right) \otimes L^{\otimes d}\right)= \\
& H^{0}\left(M, \mathcal{O}_{M}\right) \oplus H^{0}(M, L) \oplus \ldots \oplus H^{0}\left(M, L^{\otimes d}\right) .
\end{aligned}
$$

The image of $\varphi$ under this isomorphism can be decomposed as $\varphi=\left(\varphi_{0}, \ldots\right.$, $\left.\varphi_{d}\right)$ with $\varphi_{i} \in H^{0}\left(M, L^{\otimes i}\right)$ and hence

$$
\varphi=\left(\pi^{*} \varphi_{0}\right) x^{d}+\left(\pi^{*} \varphi_{1}\right) x^{d-1} y+\ldots+\left(\pi^{*} \varphi_{d}\right) y^{d},
$$

and

$$
\varphi_{\mid \operatorname{tot}(L)}=\left(\pi^{*} \varphi_{0}\right) \lambda^{d}+\left(\pi^{*} \varphi_{1}\right) \lambda^{d-1}+\ldots+\left(\pi^{*} \varphi_{d}\right) .
$$

Now the map

$$
\begin{aligned}
p_{B}: & \operatorname{tot}(L) \longrightarrow \operatorname{tot}\left(L^{\otimes d}\right) \\
\eta & \longrightarrow\left(\pi^{*} \varphi_{0}\right) \eta^{d}+\left(\pi^{*} \varphi_{1}\right) \eta^{d-1}+\ldots+\left(\pi^{*} \varphi_{d}\right)
\end{aligned}
$$

has the desired properties. 
Corollary 2.5. The above map extends to a proper map of the compactifications $p_{B}: P_{L} \rightarrow P_{L \otimes d}$. The preimage of infinite section $M_{\infty, L \otimes d}$ is a d-multiple of $M_{\infty, L}$

Proof. The extension of $p_{B}$ is given by the right hand side of (2.1).

Corollary 2.6. The map $p_{B}: \operatorname{tot}(L) \rightarrow \operatorname{tot}\left(L^{\otimes d}\right)$ is branched over a divisor $B^{\prime} \subset \operatorname{tot}\left(L^{\otimes d}\right)$ which is horizontal and of degree $d-1$ along the fibers.

Proof. From the definition of $p_{B}$ it is clear that the divisor $B^{\prime}$ is just the image under $p_{B}$ of the divisor of the section $p_{B}^{\prime}(\lambda):=d\left(\pi^{*} \varphi_{0}\right) \lambda^{d-1}+$ $(d-1)\left(\pi^{*} \varphi_{1}\right) \lambda^{d-2}+\ldots+\left(\pi^{*} \varphi_{d-1}\right) \in H^{0}\left(\operatorname{tot}(L), \pi^{*} L^{\otimes(d-1)}\right)$.

Remark 2.7. Corollary 2.6 is a complete analogue of the crucial observation of Belyi in [B].

Now we can finish the proof of theorem 2.1. Indeed let $P_{2}=\mathbb{P}\left(\mathcal{O}_{\mathbb{P}^{n-1}} \oplus\right.$ $\left.\mathcal{O}_{\mathbb{P}^{n-1}}(N)\right)$ and let $p_{B_{1}}: P_{1} \rightarrow P_{2}$ be as in corollary 2.5. Then according to corollary 2.6 the branch divisor of $p_{B_{1}} \circ f$ is the union of the zero section of $\mathcal{O}_{\mathbb{P}^{n-1}}(N)$ and a horizontal divisor $B_{2}$ of degree $N-1$ along the fibers. Now we can compose with $p_{B_{2}}+s$ where $s$ is a generic section of $\mathcal{O}_{\mathbb{P}^{n-1}}(N(N-1))$ and continue until we get a map to a $\mathbb{P}^{1}$ bundle over $\mathbb{P}^{n-1}$ branched over a union of distinct sections.

\section{The induction step}

In this section we use the induction hypothesis to modify birationally the pair $\left(P, B \cup S_{0}\right)$ obtained in Theorem 2.1 so that $B \cup S_{0}$ becomes a divisor with strict normal crossings. First we need the following proposition.

Proposition 3.1. There exists a diagram

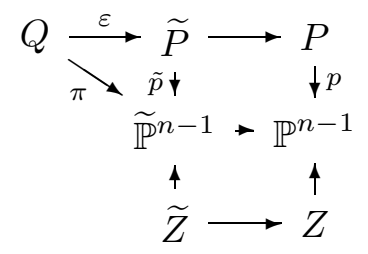

where

(i) $\widetilde{\mathbb{P}}^{n-1} \rightarrow \mathbb{P}^{n-1}$ is a birational morphism. $\widetilde{P}=\widetilde{\mathbb{P}}^{n-1} \times_{\mathbb{P}^{n-1}} P$ and $\widetilde{Z}=\widetilde{\mathbb{P}}^{n-1} \times_{\mathbb{P}^{n-1}} Z$;

(ii) $\pi$ is a flat and proper morphism whose geometric fibers are reduced and connected curves with at most ordinary double points. Furthermore $\varepsilon: Q \backslash \pi^{-1}(\widetilde{Z}) \rightarrow \widetilde{P} \backslash \tilde{p}^{-1}(\widetilde{Z})$ is an isomorphism;

(iii) $\varepsilon$ is a birational morphism which restricted on a geometric fiber $Q_{x}$ of $\pi$ falls under one of the following possibilities: 
a) $\varepsilon_{x}: Q_{x} \rightarrow \widetilde{P}_{x}$ is an isomorphism;

b) There is a (possibly disconnected) reduced rational curve $E \subset Q_{x}$ having at most ordinary double points so that $\varepsilon_{x}(E)=\Sigma$ is a finite set of closed points in $\widetilde{P}_{x}$ and

$$
\varepsilon_{x}: Q_{x} \backslash E \rightarrow \widetilde{P}_{x} \backslash \Sigma
$$

is an isomorphism;

(iii) $\widetilde{Z}$ and the preimage of $B \cup S_{0}$ in $Q$ are strict normal crossings divisors;

(iv) $Q$ is non-singular.

Proof. Denote by $Z \subset \mathbb{P}^{n-1}$ the discriminant locus of the set of sections $S_{0}, S_{1}, \ldots, S_{k+1}$. That is $Z$ is the smallest closed reduced subscheme in $\mathbb{P}^{n-1}$ whose preimage in $P$ contains all possible intersections of the $S_{i}$ 's. By induction there exists a birational morphism $\mu: \widetilde{\mathbb{P}}^{n-1} \rightarrow \mathbb{P}^{n-1}$ such that the reduced preimage $\widetilde{Z}$ of $Z$ is a divisor with strict normal crossings. Denote by $\widetilde{P}$ the fiber product of $P$ with $\widetilde{\mathbb{P}}^{n-1}$. Clearly $\widetilde{P}=\mathbb{P}\left(\mu^{*} L \oplus \mathcal{O}\right)$. Denote by $\tilde{s}_{i}, i=0, \ldots, k+1$ the sections of the projective bundle $\tilde{p}$ : $\widetilde{P} \rightarrow \widetilde{\mathbb{P}}^{n-1}$ whose images are the divisors $\widetilde{S}_{i}:=\mu^{*} S_{i}, i=0, \ldots, k+1$.

The image $\widetilde{S}_{k+1}$ of the infinity section of $\widetilde{p}$ does not intersect any of the divisors $\widetilde{S}_{i}, i=0, \ldots, k$ and thus will not cause any trouble. To deal with the bad intersections of the remaining $k+1$ sections we appeal to F. Knudsen's stabilization theorem [K, Theorem 2.4]. According to this theorem given a flat family $C \rightarrow S$ of connected reduced curves with at most ordinary double points and $a$ distinct sections $\left\{s_{i}: S \rightarrow C\right\}_{i=1}^{a}$ plus an arbitrary extra section $\Delta: S \rightarrow C$ there exist a canonical blow-up $q: C^{\prime} \rightarrow C$ and unique liftings of the sections $s_{1}, \ldots, s_{a}$ and $\Delta$ to sections $s_{1}^{\prime}, \ldots, s_{a}^{\prime}, s_{a+1}^{\prime}$ of $C^{\prime} \rightarrow S$ so that for the induced morphism $q_{s}: C_{s}^{\prime} \rightarrow C_{s}$ on any geometric fiber $C_{s}^{\prime}$ of $C^{\prime} \rightarrow S$ we have one of the following two cases

a) $q_{s}: C_{s}^{\prime} \rightarrow C_{s}$ is an isomorphism;

b) There is a rational component $E \subset C_{s}^{\prime}$ such that $s_{a+1}(s) \in E$, $q_{s}(E)=x$ is a closed point of $C_{s}$ and

$$
q_{s}: C_{s}^{\prime} \backslash E \longrightarrow C_{s} \backslash\{x\}
$$

is an isomorphism.

In particular, $C^{\prime} \rightarrow S$ is again a flat family of reduced curves with at most ordinary double points. Explicitly $q: C^{\prime} \rightarrow C$ is given as follows. Denote by $\mathcal{J}$ the $\mathcal{O}_{C}$ ideal defining $\Delta$ and by $\mathcal{C}$ the cokernel of the diagonal embedding

$$
\mathcal{O}_{C} \longrightarrow \mathcal{J}^{\vee} \oplus \mathcal{O}_{C}\left(s_{1}+\ldots+s_{a}\right)
$$


Then

$$
C^{\prime}:=\operatorname{Proj}\left(\oplus_{i \geq 0} \mathcal{C}^{i}\right),
$$

and $q: C^{\prime} \rightarrow C$ is the natural structure morphism.

The total space of $C^{\prime}$ can be made smooth by a sequence of blow-ups with smooth centers as long as $C$ and $S$ are smooth and the morphism $C \rightarrow S$ is smooth in the complement of a divisor with strict normal crossings. This can be seen directly by analyzing the local picture around the separated sections but instead of doing that we prefer to invoke a much stronger general result of A.J. de Jong. The [dJ, Proposition 5.6] guarantees that the total space of any split flat family of semistable curves over a smooth base which is smooth over the complement of a strict normal crossings divisor can be blown-up so that the resulting family is of the same type and has a smooth total space. Thus the only thing that requires checking is that the family $C^{\prime}$ is split but this is clear since we can label the components of every fiber of $C^{\prime}$ by their distance from the proper transform of the fiber in $C$.

Remark 3.2. The stabilization theorem is stated in $[\mathrm{K}]$ for families of stable curves since Knudsen is interested in obtaining a morphism between the universal curve over the stack of $n$-pointed curves and the stack of $n+1$ pointed curves. However the proof he gives in $[\mathrm{K}]$ is carefully designed to work for general flat families of reduced nodal curves. In particular, the stability assumption is never used in his argument.

Applying the stabilization theorem to each of the sections $\widetilde{S}_{1}, \ldots \widetilde{S}_{k}$ (or rather to the corresponding proper transforms) in turn, we obtain a variety $Q$ together with a birational morphism $\varepsilon: Q \rightarrow \widetilde{P}$ and $k+2$ distinct sections $t_{i}: \widetilde{\mathbb{P}}^{n-1} \rightarrow Q, i=0, \ldots, k+1$ lifting the sections $\tilde{s}_{i}$. Since at every step we are blowing-up only ideal sheaves whose support is contained in the preimage of $\widetilde{Z}$ the construction of $Q$ guarantees the validity of items (ii) and (iii) of the statement of the proposition. Furthermore due to part b) of the stabilization theorem the images of the sections $t_{i}$ intersect every fiber of $\pi$ at a smooth point. Thus the preimage of $B \cup S_{0}$ in $Q$ is the same as the union of the images of the $t_{i}$ 's and $\pi^{-1}(\widetilde{Z})$. Since $\pi$ is flat and has singular fibers that are trees of rational curves we conclude that $\pi^{-1}(\widetilde{Z})$ is also a divisor with strict normal crossings which finishes the proof of the proposition.

To finish the proof of Theorem A consider the normalization $Y$ of the fiber product $X \times{ }_{P} Q$. Denote by $Y^{\circ}$ the preimage of $Q \backslash\left\{B \cup S_{0}\right\}$ in $Y$. The normal variety $Y$ is a finite cover of the smooth variety $Q$ branched along a divisor with strict normal crossings. Since we are in characteristic zero this implies that $Y$ has only abelian quotient singularities and is toroidal without self-intersections, i.e. locally in the étale topology (or formally) the 
embedding $Y^{\circ} \subset Y$ is isomorphic to the embedding of an affine algebraic torus $U$ in its toric variety $\bar{U}$ so that every component of the complement $\bar{U} \backslash U$ is normal. Finally we invoke the fundamental theorem [KKMS, Ch. II, $\S 2$, Theorem $11 *]$ about toric resolutions according to which there exists a canonical sheaf of ideals on $I \subset \mathcal{O}_{Y}$ such that the blow-up $B l_{I}(Y)$ is non-singular.

\section{Acknowledgements}

We would like to thank A.J. de Jong for his inspiring Santa Cruz lectures and D. Abramovich for suggesting that we use semistable reduction for pointed curves of genus zero which allowed us to substantially simplify our original application of the induction. We would also like to thank the referee for the careful reading of the manuscript and for the many valuable comments.

\section{References}

[A-dJ] D. Abramovich and A. J. de Jong, Smoothness, semistability and toroidal geometry, preprint, alg-geom/9603018.

[B] G. V. Belyi, Galois extensions of a maximal cyclotomic field, Izv. Akad. Nauk SSSR Ser. Mat. 43, no. 2 (1979), 267-276.

[B-M] E. Bierstone and P. Milman, Canonical desingularization in characteristic zero by blowing up the maximum strata of a local invariant, preprint, alggeom/9508005.

[F-H] W. Fulton and J. Hansen, A connectedness theorem for projective varieties, with applications to intersection and singularities of mappings, Ann. of Math. 110 (1979), 159-166.

[Ha] R. Hartshorne, Algebraic geometry, Springer, 1977.

[Hi] H. Hironaka, Resolution of singularities of an algebraic variety over a field of characteristic zero: I, II, Ann. of Math. (2) 79 (1964), 109-326.

[dJ] A. J. de Jong, Smoothness, semistability and alterations, preprint, http://www.math.uiuc. edu/K-theory/0081/alterations.dvi

[KKMS] G. Kempf, F. Knudsen, D. Mumford, and B. Saint-Donat, Toroidal embeddings I, LNM no 339, Springer-Verlag, 1973.

[K] F. Knudsen, The projectivity of the moduli space of stable curves, II, Math. Scand. 52 (1983), 161-199.

[SGA 1] A. Grothendieck et al., Séminaire de Géométrie Algebrique du Bois Maire 1960/61, Lecture Notes in Math. 224, Springer-Verlag.

[S] M. Spivakovsky, Resolution of singularities, preprint, 1994.

Courant Institute for Mathematical Sciences, New York University, New York, NY 10012-1110 and Steklov Institute of Mathematics, Russian Academy of Sciences, 42 Vavilova street, Moscow 117333, Russia

E-mail address: bogomolo@cims.nyu.edu

Department of Mathematics, Massachusetts Institute of Technology, CamBRIDGE, MA 02139-4307

E-mail address: pantev@math.mit.edu 